\title{
Review: several diagnostic aids have moderate to high accuracy for detecting abnormalities in acute knee pain
}

Jackson JL, O'Malley PG, Kroenke K. Evaluation of acute knee pain in primary care. Ann Intern Med 2003;139:575-88.

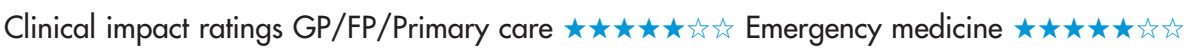

What is the accuracy of clinical decision rules (CDR), physical examination (PE), and imaging procedures (eg, magnetic resonance imaging [MRI]) for detecting causes of acute knee pain?

\section{METHODS}

Data sources: Medline (1966 to October 2002) and
bibliographies of relevant articles.

\section{MAIN RESULTS}

(1) CDR for ruling out knee fractures. Of the 4 CDR evaluated ( 1 study each), the Ottawa knee rules had the most thorough validation (sensitivity 100\%,95\% CI 94 to 100; specificity $49 \%$, CI 46 to 52 ) for detecting knee fractures. (2) PE and MRI for detecting knee cartilage and ligament injuries. 35 and 89 studies that evaluated PE and MRI, respectively, met the selection criteria. The table shows the results. (3) Osteoarthritis as a differential diagnosis in acute knee pain. The presence of $\geqslant 4$ of 6 American College of Rheumatology clinical criteria (age $>50 \mathrm{y}$, stiffness for $<30$ minutes, crepitus, bony tenderness, bony enlargement, and no palpable warmth) for detecting osteoarthritis had a sensitivity of $84 \%$ and specificity $89 \%$. Presence of osteophytes on plain radiographs plus $\geqslant 1$ of age $>50$ years, crepitus, or morning stiffness of $\leqslant 30$ minutes increased sensitivity to $91 \%$ with a specificity of $86 \%$.

\section{CONCLUSIONS}

In patients with acute knee pain, the Ottawa knee rules have 100\% sensitivity but low specificity for detecting fractures of the knee;

For correspondence: Dr J $\mathrm{Z}$ Jackson, Uniformed Services University of the Health Sciences, Bethesda, MD, USA. jejackson@usuhs.mil

Source of funding: not stated. physical examination and magnetic resonance imaging have moderate to high sensitivity and specificity for detecting meniscal or ligamentous injuries; and clinical criteria have moderate to high sensitivity and specificity for detecting osteoarthritis.

\section{Commentary}

$\mathrm{T}$

he review by Jackson et al emphasizes the importance of using CDR, $\mathrm{PE}$, and clinical criteria to arrive at a diagnosis for causes of acute knee pain without relying on diagnostic tests such as $x$ rays or MRI Summary values of sensitivity were estimated from fitted receiver operating characteristics (ROC) curves corresponding to the median specificity of the observed data. This method does not rely on tests of heterogeneity and clearly has advantages over weighting the sensitivity and specificity of each study when evidence of heterogeneity exists. Furthermore, the authors appear to have reviewed a large selection of studies.

However, the methodology of this review has some limitations. Firstly, only a single database (Medline) was used as the data source. Secondly, despite the advantages of using fitted ROC curves, the original studies may have been too heterogenous in terms of the "cut off" points for positive results or the type of outcomes they used (eg, range of sensitivity 26-93\% and specificity 22-100\% for PE); making any attempt at quantitative synthesis of the data seem unreasonable. Thirdly, a weakness inherent to all original studies that evaluated PE was partial blinding larthroscopy-the gold standard was done by orthopaedists who knew results of the PE).

Using the Ottawa knee rule, physicians may decrease the use of $x$ rays by $25 \%$ for acute knee injuries. ${ }^{1}$ Training family physicians to become better at $\mathrm{PE}$ of the knee may reduce the number of unnecessary imaging investigations ordered because the PE tests are quite accurate. Using clinical criteria is also an accurate tool in diagnosing osteoarthritis.

$$
\begin{array}{r}
\text { Ken Trinh, MD, BSc, MSc, PhD (Can) } \\
\text { McMaster University }
\end{array}
$$
Hamilton, Ontario, Canada

1 Stiell IG, Wells GA, Hoag RH, et al. Implementation of the OHtawa Knee Rule for the use of radiography in acute knee injuries. JAMA 1997;278:2075-9.

Diagnostic characteristics of physical examination (PE) and magnetic resonance imaging (MRI) for detecting meniscal, ligamentous, and cartilage tears in acute knee pain*

\begin{tabular}{llllrr}
\hline Test & Characteristic & Sensitivity (95\% CI) & Specificity (CI) & +LR & - LR \\
\hline PE & Medial meniscus & $86 \%(79$ to 92$)$ & $72 \%(61$ to 83$)$ & 3.1 & 0.19 \\
& Lateral meniscus & $88 \%(77$ to 99$)$ & $92 \%(89$ to 95$)$ & 11.0 & 0.13 \\
& Anterior cruciate ligament tear & $74 \%(60$ to 88$)$ & $95 \%(92$ to 98$)$ & 15.0 & 0.27 \\
& Posterior cruciate ligament tear & $81 \%(63$ to 98$)$ & $95 \%(81$ to 100$)$ & 16.0 & 0.20 \\
& Cartilage injury & $51 \%(37$ to 65$)$ & $96 \%(91$ to 100$)$ & 13.0 & 0.51 \\
\multirow{3}{*}{ MRI } & Medial meniscus & $89 \%(83$ to 95$)$ & $80 \%$ (73 to 87$)$ & 4.5 & 0.14 \\
& Lateral meniscus & $79 \%(73$ to 85$)$ & $91 \%(84$ to 98$)$ & 8.7 & 0.23 \\
& Anterior cruciate ligament tear & $87 \%(83$ to 91$)$ & $91 \%(88$ to 94$)$ & 9.6 & 0.14 \\
& Posterior cruciate ligament tear & $75 \%(65$ to 85$)$ & $93 \%(88$ to 98$)$ & 11 & 0.27 \\
& Cartilage injury & $84 \%(67$ to 100$)$ & $90 \%(85$ to 96$)$ & 8.4 & 0.17 \\
\hline
\end{tabular}

*Diagnostic terms defined in glossary. 